\title{
Experimental characterization of the outdoor MIMO wireless channel temporal variation
}

\author{
Michael A. Jensen \\ jensen@byu.edu \\ Karl F. Warnick \\ warnick@byu.edu \\ Jon W. Wallace \\ wall@ieee.org \\ Ajay Gummalla \\ Harry B. Lee
}

Follow this and additional works at: https://scholarsarchive.byu.edu/facpub

Part of the Electrical and Computer Engineering Commons

\section{Original Publication Citation}

Wallace, J. W., et al. "Experimental Characterization of the Outdoor MIMO Wireless Channel Temporal Variation." Vehicular Technology, IEEE Transactions on 56.3 (27): 141-9

\section{BYU ScholarsArchive Citation}

Jensen, Michael A.; Warnick, Karl F.; Wallace, Jon W.; Gummalla, Ajay; and Lee, Harry B., "Experimental characterization of the outdoor MIMO wireless channel temporal variation" (2007). Faculty Publications. 257.

https://scholarsarchive.byu.edu/facpub/257

This Peer-Reviewed Article is brought to you for free and open access by BYU ScholarsArchive. It has been accepted for inclusion in Faculty Publications by an authorized administrator of BYU ScholarsArchive. For more information, please contact ellen_amatangelo@byu.edu. 


\title{
Experimental Characterization of the Outdoor MIMO Wireless Channel Temporal Variation
}

\author{
Jon W. Wallace, Member, IEEE, Michael A. Jensen, Senior Member, IEEE, \\ Ajay Gummalla, Member, IEEE, and Harry B. Lee, Member, IEEE
}

\begin{abstract}
Time-variant multiple-input multiple-output (MIMO) channels are measured in an outdoor campus environment at $2.45 \mathrm{GHz}$ with directional patch arrays and omnidirectional monopole arrays. A number of useful metrics are proposed for quantifying time variation in MIMO channels: eigenvalue level crossing rate, eigenvector angular deviation, and capacity loss for delayed transmit and receive channel state information (CSI). Measurements in four different environments confirm the strong correlation between angular spread of multipath and MIMO channel time variability. The rate of time variation is also strongly influenced by the type of array, indicating that directional elements may be advantageous for highly mobile environments. The proposed metrics indicate that although the physical communication layer may need to update CSI several times per wavelength, the required rate of adaptation in transmit rate, modulation, and power allocation is much less severe.
\end{abstract}

Index Terms-Information theory, multiple-input multipleoutput (MIMO) systems, time-varying channels.

\section{INTRODUCTION}

A NALYTICAL studies and measurement campaigns have demonstrated the dramatic capacity increase enabled by exploiting the multipath spatial structure with multipleinput multiple-output (MIMO) communications [1], [2]. However, realization of these gains depends critically on the availability of channel state information (CSI) [3], which is typically obtained by periodically transmitting known training sequences. When the channel varies rapidly, the required frequency of training can diminish and eventually offset the capacity improvement enabled by MIMO technology. Although signaling strategies for rapidly varying MIMO channels exist

Manuscript received January 4, 2006; revised May 19, 2006 and July 5, 2006. This work was supported in part by the Defense Advanced Research Projects Agency under Phase 2 SBIR Award \#HR0011-04-C-0029, by the National Science Foundation under Information Technology Grants CCR-0313056 and CCF-0428004, and by the U.S. Army Research Office under the MultiUniversity Research Initiative Grant W911NF-04-1-0224. The review of this paper was coordinated by Dr. K. Dandekar.

J. W. Wallace was with the Department of Electrical and Computer Engineering, Brigham Young University, Provo, UT 84602 USA. He is now with the School of Engineering and Science, Jacobs University Bremen (formerly International University of Bremen), 28725 Bremen, Germany (e-mail: wall@ieee.org).

M. A. Jensen is with Brigham Young University, Provo, UT 84602 USA (e-mail: jensen@ee.byu.edu).

A. Gummalla was with San Diego Research Center, Inc., San Diego, CA 92121-2950 USA. He is now with Rayspan Corporation, San Diego, CA 921302543 USA (e-mail: ajayg@ieee.org).

H. B. Lee is with San Diego Research Center, Inc., San Diego, CA 921212950 USA.

Digital Object Identifier 10.1109/TVT.2007.895599
[4], such methods typically provide modest capacity increase relative to the gains associated with methods exploiting full CSI [5], [6].

Since the rate of time variation effectively limits the achievable MIMO capacity, an understanding of the degree of time variation in real-world channels is very important. Such knowledge helps in determining the type of MIMO technology to apply for specific applications. Previously reported MIMO measurements have shown that a moving person can temporarily inhibit a single indoor channel eigenmode [7], and that the coherence time of outdoor channels is about twice that predicted by Jakes' model [8]. Furthermore, recent work demonstrates the effect of delayed receive CSI on the bit error rate of MIMO systems in an indoor environment [9]. However, to date there does not appear to be a comprehensive analysis providing metrics for MIMO channel variation combined with real-world channel measurements with the goal of demonstrating how the variation impacts performance in practice. Also, the effect of directional and dual-polarization elements on channel time variation has received little attention.

In this paper, we evaluate the channel time variation from narrowband measurements taken at $2.45 \mathrm{GHz}$ in several representative outdoor locations. Instead of simply plotting eigenvalues of time-varying channels or applying previous single-input single-output (SISO) metrics, we present true MIMO metrics that quantify the rate of channel time variation, thus allowing the channels to be classified based on their time variability. Combining outdoor measurements with useful time-variation metrics illustrates general limitations imposed by time-varying channels and serves as a benchmark for later studies.

\section{NARrowband MiMO Measurement System}

In this section, we briefly describe the MIMO channel sounder employed, with an emphasis on parameters specific to this measurement campaign. We refer the reader to [10] for additional details on the measurement system.

\section{A. Channel Sounder}

Figs. 1 and 2 depict a high-level block diagram of the narrowband MIMO channel sounder and the actual transmit/receive subsystems, respectively. The channel is probed by transmitting a high-frequency carrier on $N_{T}$ different transmit antennas, each modulated with an independent code word. The receiver simultaneously samples the intermediate frequency waveforms 


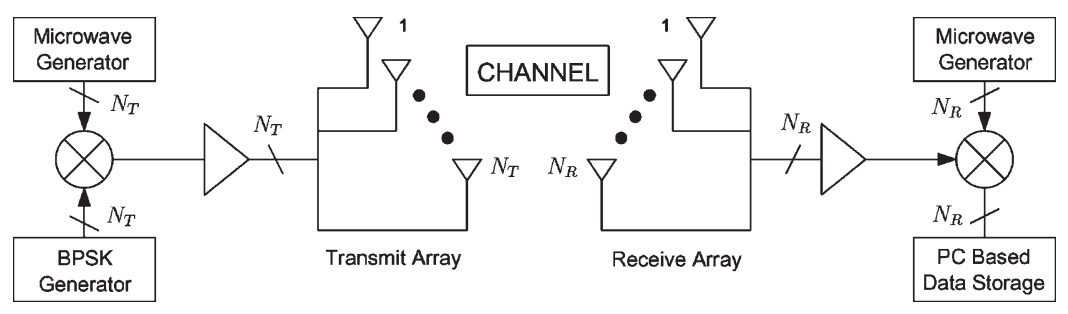

Fig. 1. High-level block diagram of narrowband MIMO channel sounder used in this paper.
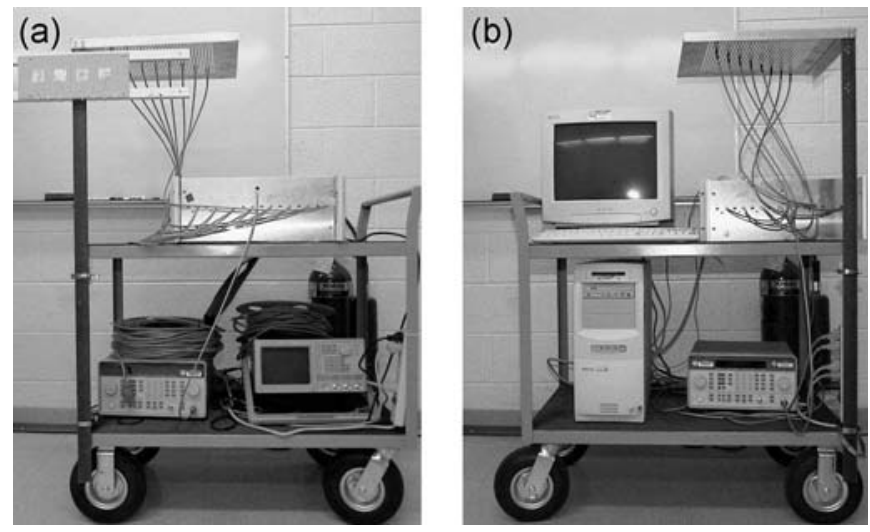

Fig. 2. Photographs of (a) transmit and (b) receive subsystems. Equipment is placed on carts for mobile outdoor measurements.

TABLE I

Measurement Parameters for Measurement Campaign

\begin{tabular}{|l|l|}
\hline Parameter & Value \\
\hline Carrier Frequency $\left(f_{C}\right)$ & $2.45 \mathrm{GHz}$ \\
Bandwidth $(W)$ & $30 \mathrm{kHz}$ \\
Transmit Power $\left(P_{T}\right)$ & $0.5 \mathrm{~W}$ per Channel \\
Code Rate $(R)$ & $12.5 \mathrm{kS} / \mathrm{s}$ \\
Intermediate Frequency $\left(f_{\mathrm{IF}}\right)$ & $25 \mathrm{kHz}$ \\
Codewords & $16 \mathrm{~b} \mathrm{Walsh}+15 \mathrm{~b}$ Alignment \\
Temporal Resolution $(\Delta t)$ & $2.5 \mathrm{~ms} / 25 \mathrm{~ms}$ \\
\hline
\end{tabular}

received on $N_{R}$ different receive antennas, thus allowing the formation of an $N_{R} \times N_{T}$ narrowband channel matrix.

\section{B. Measurement Parameters}

Table I lists the important parameters of the system for this measurement campaign common to all measurement locations. The nominal $30-\mathrm{kHz}$ bandwidth allowed a single tone in the 2.4-GHz industrial, scientific, and medical band to be measured. Each transmit channel used a repeated 31-bit sequence consisting of a unique 16-bit Walsh code combined with a common 15-bit alignment code. The alignment code was chosen to have favorable autocorrelation properties, allowing correct alignment of the waveforms to be achieved in postprocessing. Although the system produced channel snapshots for each $2.5 \mathrm{~ms}$ of measurement time, the data were smoothed by a factor of 10 in time to improve signal-to-noise ratio (SNR).

\section{Antenna Arrays}

Fig. 3 depicts the two basic array types used in this paper, namely: 1) a uniform linear array (ULA) of eight vertically polarized monopole antennas and 2) a linear array of four dualpolarized patch antennas. The monopole antennas of length
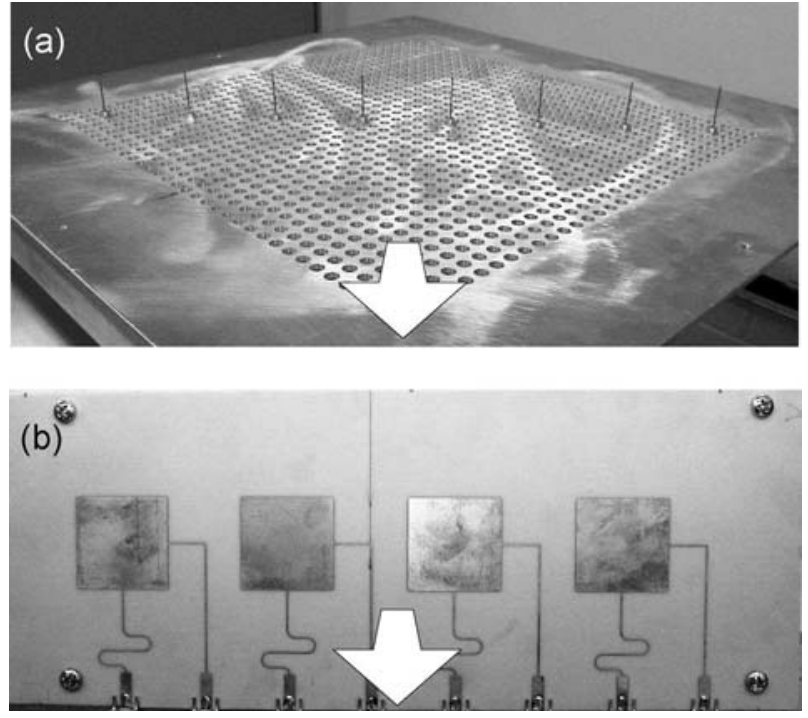

Fig. 3. Two types of antenna arrays employed in this paper. (a) $\lambda / 4$ monopole antennas mounted on a reconfigurable ground plane. (b) Dual-polarization patch antennas. Arrows pointing out of the page indicate orientation direction for the two arrays.

$\lambda / 4$, where $\lambda$ is the free space wavelength, exhibit nearly uniform radiation patterns in the azimuthal plane. Each patch element has two independent feeds for vertical and horizontal polarizations, with the polarizations exhibiting 3-dB azimuthal beamwidths of approximately $90^{\circ}$ and $120^{\circ}$, respectively. The use of the two array types allows the investigation of the effect of directivity and polarization on the rate of channel variation.

\section{Channel Metrics}

An important part of this paper is the identification of metrics that quantify the time variation of the measured channel responses. Each channel metric can be computed from the channel transfer matrix elements $H_{i j}^{(n)}$, where $n$ is a channel measurement time index, and $i$ and $j$ are the receive and transmit antenna indices, respectively. Since the temporal variation of measured channels results largely from receiver movement, time-variation metrics are given in terms of distance, which can readily be converted to time given a receiver motion velocity.

Many of the metrics presented make use of the notion of parallel spatial channels enabled by the array and the multipath propagation. As way of background, let the singular value decomposition of the channel matrix at time index $n$ be given as $\mathbf{H}^{(n)}=\mathbf{U}^{(n)} \mathbf{S}^{(n)} \mathbf{V}^{(n) H}$, where $\mathbf{U}^{(n)}$ and $\mathbf{V}^{(n)}$ are unitary matrices of singular vectors, $\mathbf{S}^{(n)}$ is a diagonal matrix of real singular values, and $\{\cdot\}^{H}$ is the Hermitian operator. If we precode the vector $\mathbf{x}_{0}^{(n)}$ of transmit symbols by the right singular 
vectors using $\mathbf{x}^{(n)}=\mathbf{V}^{(n)} \mathbf{x}_{0}^{(n)}$ and weight the resulting received signal vector by the left singular vectors, we obtain

$$
\begin{aligned}
\mathbf{y}_{0}^{(n)} & =\mathbf{U}^{(n) H} \mathbf{y}^{(n)} \\
& =\mathbf{U}^{(n) H} \mathbf{H}^{(n)} \mathbf{x}^{(n)}+\mathbf{U}^{(n) H} \boldsymbol{\eta}^{(n)} \\
& =\mathbf{S}^{(n)} \mathbf{x}_{0}^{(n)}+\boldsymbol{\eta}_{0}^{(n)}
\end{aligned}
$$

where $\boldsymbol{\eta}^{(n)}$ is the noise at the $n$th time index. Throughout this discussion, it will be assumed that the noise vector consists of zero-mean Gaussian random variables and has covariance $\sigma^{2} \mathbf{I}$, where $\mathbf{I}$ is the identity matrix. Since the received signal vector is now a scaled (and noisy) version of the transmitted vector, this weighting effectively creates a set of independent spatial modes over which the data are communicated. In the following, we will refer to the $i$ th columns of $\mathbf{U}^{(n)}$ and $\mathbf{V}^{(n)}$, symbolized by $\mathbf{u}_{i}^{(n)}$ and $\mathbf{v}_{i}^{(n)}$, as the $i$ th receive and transmit eigenvectors, respectively, and $\gamma_{i}^{(n)}=S_{i i}^{(n) 2}$ as the $i$ th channel eigenvalue.

\section{A. Capacity Metrics}

The Shannon capacity, which is the upper bound of achievable rates for error-free transmission, is a key figure of merit for MIMO channels. In this paper, we consider narrowband MIMO capacity under the conditions where the transmitter is informed and uninformed about the CSI. All numerical values of capacity are given in terms of bits per second per hertz. Note that since this paper analyzes time-variant channels, and notions of capacity usually assume an infinite time window for coding, the time-variant capacity in our context represents a figure of merit as opposed to a truly achievable capacity. These values serve as a bound that becomes tight as either the velocity vanishes or the symbol rate grows large.

1) Informed Transmit Capacity $C_{\mathrm{WF}}$ : A transmitter with perfect CSI may diagonalize the channel as outlined above and subsequently use water-filling on the parallel Gaussian channels to obtain the capacity, i.e.,

$$
\begin{aligned}
C_{\mathrm{WF}}^{(n)} & =\sum_{i} \log _{2}\left(1+\frac{p_{i}^{(n)} \gamma_{i}^{(n)}}{\sigma^{2}}\right) \\
p_{i}^{(n)} & =\left(\nu-\sigma^{2} / \gamma_{i}^{(n)}\right)^{+} \\
(z)^{+} & = \begin{cases}z, & z \geq 0 \\
0, & \text { otherwise }\end{cases}
\end{aligned}
$$

where $p_{i}$ is the power delivered to the $i$ th parallel channel, $\nu$ is determined using the constraint $\sum_{i} p_{i}^{(n)}=P_{T}$, and $P_{T}$ is the total transmit power. Typically, $\mathbf{H}^{(n)}, \sigma^{2}$, and $P_{T}$ are scaled to obtain a prescribed average SISO SNR that is reasonable for a realistic system. In this paper, a SISO SNR of $10 \mathrm{~dB}$ was assumed for all capacity computations.

2) Uninformed Transmit Capacity $C_{\mathrm{UT}}$ : When the transmitter has no knowledge about channel state, rank, or statistics, the best strategy involves delivering equal power in independent streams to the transmit antennas. In this case, the channel capacity is given by

$$
C_{\mathrm{UT}}^{(n)}=\log _{2}\left|\frac{P_{T} \mathbf{H}^{(n)} \mathbf{H}^{(n) H}}{N_{T} \sigma^{2}}+\mathbf{I}\right| .
$$

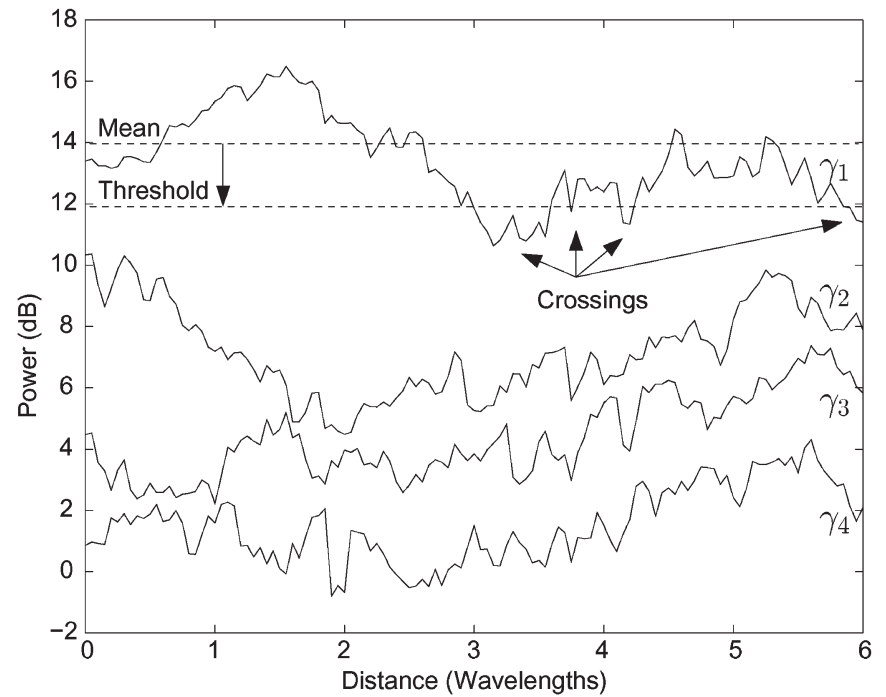

Fig. 4. Example plot of the time variation of the first four channel eigenvalues, illustrating the meaning of the ELCR metric.

\section{B. Eigenchannel Metrics}

Since achieving capacity involves transmitting independent information on the parallel channel eigenmodes, it is interesting to study the temporal behavior of these modes. The following metrics represent possible mechanisms for quantifying the temporal variability of the channel eigenvalues and eigenvectors.

1) Eigenvalue Level Crossing Rate (ELCR): $\mathrm{ELCR}_{i}$ is the number of times $\gamma_{i}^{(n)}$, which represents the power gain of the $i$ th eigenmode, drops below a specified threshold divided by the total distance traveled. This concept is illustrated in Fig. 4. In this paper, a threshold of $2 \mathrm{~dB}$ below the mean is assumed, and ELCR is specified as the average number of crossings per wavelength.

ELCR is interesting from the point of view of an adaptive MIMO physical layer (PHY) and a medium access layer (MAC) that must adapt transmission rate and modulation to the timedependent channel quality. This metric also indicates the level of coding that may be required to overcome channel fades for constant rate/modulation transmission.

2) Eigenvector Angular Deviation (EAD): EAD quantifies how quickly the transmit and receive eigenvectors rotate in complex multidimensional space. We define EAD for the transmit space as

$$
\theta_{i k}=\frac{1}{N-k} \sum_{n=1}^{N-k} \cos ^{-1}\left|\mathbf{v}_{i}^{(n) H} \mathbf{v}_{i}^{(n+k)}\right|
$$

where $k$ is the distance between two channel snapshots, and $N$ is the total number of snapshots, with an analogous definition for the receiver EAD. EAD directly impacts how quickly the PHY must update transmission weights to track the time-variant MIMO channel. However, since the information in this metric is somewhat redundant with the information provided by the capacity degradation metrics in Section III-C, it will not be used in the following data analysis. 
3) Eigenvalue Spread (ES): ES indicates the amount of multipath in the channel, ranging from large values for nearly line-of-sight (LOS) channels to lower values for channels with richer multipath. ES in this campaign is defined as $\mathrm{ES}=$ $10 \log _{10}\left(\bar{\gamma}_{1}\right)-10 \log _{10}\left(\bar{\gamma}_{3}\right)$, where $\bar{\gamma}_{i}$ is the mean of the $i$ th eigenvalue.

\section{Capacity Degradation Metrics}

Although the eigenchannel metrics are useful for system specification and design, they do not indicate the loss of channel quality in an information-theoretic sense. Furthermore, the time-variant capacity metrics only provide an instantaneous measure of capacity (within the limits outlined in Section III-A). Here, we define simple metrics for quantifying the loss in capacity due to channel time variation.

1) Transmit CSI Delay: First, consider the case where the receiver has perfect CSI but the transmitter only has the delayed channel estimate $\widehat{\mathbf{H}}$. We may define capacity for delayed transmit CSI as

$$
C_{T}^{(n)}=\log _{2}\left|\frac{\mathbf{H}^{(n)} \mathbf{Q}(\widehat{\mathbf{H}}) \mathbf{H}^{(n) H}}{\sigma^{2}}+\mathbf{I}\right|
$$

where $\mathbf{H}$ is the true channel, $\sigma^{2}$ is the receiver noise variance, $\mathbf{Q}(\widehat{\mathbf{H}})$ is the optimal transmit covariance given by the water-filling solution (assuming $\widehat{\mathbf{H}}$ represents the true channel), $\operatorname{Tr}\{\mathbf{Q}\} \leq P_{T}$, and $P_{T}$ is total transmit power. As the estimate $\widehat{\mathbf{H}}$ becomes increasingly outdated, $C_{T}^{(n)}$ will tend to decrease. When $C_{T}^{(n)}$ falls below the uninformed transmit capacity $\left(C_{T}^{(n)}\right.$ with $\mathbf{Q}=\mathbf{I}$ ), which occurs at the motion distance $d_{T}$, the transmit CSI is no longer useful.

2) Receive CSI Delay (RCD): Next, consider the case where both transmitter and receiver have outdated CSI. With imperfect channel estimates $\widehat{\mathbf{H}}=\widehat{\mathbf{U}} \widehat{\mathbf{S}}^{H}$, we can rearrange the received signal as

$$
\mathbf{y}^{(n)}=\widehat{\mathbf{H}} \mathbf{x}^{(n)}+\left[\mathbf{H}^{(n)}-\widehat{\mathbf{H}}\right] \mathbf{x}^{(n)}+\boldsymbol{\eta}^{(n)}
$$

where $\mathbf{x}^{(n)}=\widehat{\mathbf{V}} \mathbf{x}_{0}^{(n)}$. Detection of the received waveform using the outdated CSI leads to a modification of (1) given by

$$
\widehat{\mathbf{y}}_{0}^{(n)}=\widehat{\mathbf{U}}^{H} \mathbf{y}^{(n)}=\widehat{\mathbf{S}} \mathbf{x}_{0}^{(n)}+\mathbf{M}^{(n)} \mathbf{x}_{0}^{(n)}+\widehat{\mathbf{U}}^{H} \boldsymbol{\eta}^{(n)}
$$

where $\mathbf{M}^{(n)}=\widehat{\mathbf{U}}^{H}\left[\mathbf{H}^{(n)}-\widehat{\mathbf{H}}\right] \widehat{\mathbf{V}}$. This procedure therefore constructs parallel channels with gains $\hat{S}_{i i}$ but with selfinterference (or "crosstalk") controlled by the matrix $\mathbf{M}^{(n)}$.

We make no assumptions about the distribution of the channel, which in turn leads to unknown statistics for $\mathbf{M}^{(n)}$. Unfortunately, defining the capacity of this channel rigorously is difficult, and we therefore construct a lower bound for the capacity by computing the mutual information of a simplified system. Specifically, it is realistic to assume that the receiver knows the level of self-interference on the parallel subchannels but is unaware of the cross correlation. Mathematically, we assume the interference vector $\mathbf{z}^{(n)}=\mathbf{M}^{(n)} \mathbf{x}_{0}^{(n)}$ consists of independent zero-mean Gaussian elements with variance (at time sample $n$ ) of $\left\{\mathbf{R}_{z}^{(n)}\right\}_{i i}=\left\{\mathbf{M}^{(n)} \mathbf{R}_{x}^{(n)} \mathbf{M}^{(n) H}\right\}_{i i}$, where $\mathbf{R}_{x}^{(n)}$ is the covariance of $\mathbf{x}_{0}^{(n)}$. The mutual information of this system is

$$
\begin{aligned}
C_{R}^{(n)} & =\sum_{i} \log _{2}\left(1+p_{i}^{(n)} \gamma_{i}^{(n)} / q_{i}^{(n)}\right) \\
q_{i}^{(n)} & =\left\{\mathbf{M}^{(n)} \mathbf{R}_{x}^{(n)} \mathbf{M}^{(n) H}\right\}_{i i}+\sigma^{2}
\end{aligned}
$$

where $\mathbf{R}_{x}^{(n)}=\operatorname{diag}\left(\mathbf{p}^{(n)}\right)$ with $p_{i}^{(n)}$ found according to waterfilling (assuming $\mathbf{H}^{(n)}=\widehat{\mathbf{H}}$ and $q_{i}^{(n)}=\sigma^{2}$ ). We define $d_{R}$ as the distance at which $C_{R}$ drops to $50 \%$ of its maximum value. We note that this RCD capacity is very similar to the capacity defined in [11], which has been applied to ray-tracing simulations of time-varying urban channels.

Since the capacity degradation metrics are a function of delay between the points in time when the channel CSI is obtained and eventually used, these metrics may be averaged over many starting points to obtain a more general understanding of the effect of time variation on capacity.

\section{Spatial Spectra}

It is intuitive that the rate of channel time variation is linked to the angle spread of the multipath field, motivating a study of the channel spatial structure. The channel spatial spectrum represents the relative power transfer through the channel as a function of transmit or receive angle. A simple Bartlett (or Fourier) beamformer estimates the transmit and receive spectra as

$$
\begin{aligned}
P(\phi) & =\mathbf{a}^{H}(\phi) \mathbf{R} \mathbf{a}(\phi) \\
a_{i}(\phi) & =f_{i}(\phi) \exp \left[j k_{0}\left(x_{i} \cos \phi+y_{i} \sin \phi\right)\right]
\end{aligned}
$$

where $\mathbf{a}$ is the array steering vector, $\mathbf{R}$ is a transmit or receive covariance matrix, $f_{i}(\phi)$ is the far-field radiation/reception pattern of the $i$ th antenna in the horizontal plane, $k_{0}$ is the freespace wavenumber, and $x_{i}$ and $y_{i}$ are the coordinates of the $i$ th antenna. Covariance matrices for transmit and receive are, respectively, estimated using

$$
\begin{aligned}
R_{T, j_{1}, j_{2}} & =\frac{1}{N N_{R}} \sum_{i=1}^{N_{R}} \sum_{n=1}^{N} H_{i j_{1}}^{(n)} H_{i j_{2}}^{(n) *} \\
R_{R, i_{1}, i_{2}} & =\frac{1}{N N_{T}} \sum_{j=1}^{N_{T}} \sum_{n=1}^{N} H_{i_{1} j}^{(n)} H_{i_{2} j}^{(n) *} .
\end{aligned}
$$

\section{Channel Measurements}

Measurements were taken in four different environments. In all cases, the transmitter remained stationary during the measurement time. In the following, the designation 8P refers to the eight-port (four-element) dual-polarized patch array with an element spacing of $0.5 \lambda$. The designation $n \mathrm{M}$ refers to the $n$-element monopole ULA, where the interelement spacing is $0.44 \lambda$ unless otherwise noted. 


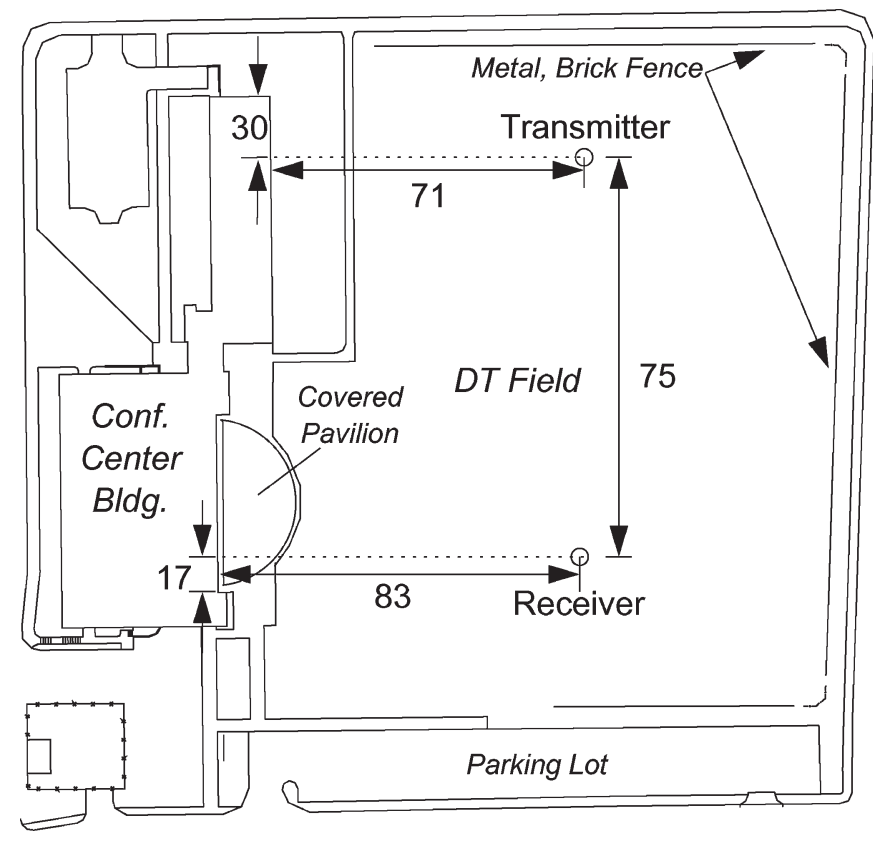

Fig. 5. Map of Environment 1 (DT field). Transmitter and receiver were placed in a large open field to the east of a single large building, simulating a low-scattering environment. Distances are in meters.

\section{A. Deseret Towers (DT) Field}

Initial measurements were taken at the DT field, which is a large open area, surrounded by a 1.5-m-high fence composed of vertical metal rods and bricks, with a single large building nearby. The transmitter and receiver were placed about $70 \mathrm{~m}$ to the east of the building, as shown in Fig. 5. The monopole array spacing was set to $0.4 \lambda$ for these experiments. Furthermore, a reflecting plate was placed on the side of the monopole transmit array to block the LOS component.

Two experiments were run to determine a suitable spatial sample rate for the moving receiver. The receiver first traveled approximately $2.5 \mathrm{~cm} / \mathrm{s}$ over a distance of $75 \mathrm{~cm}$, with a sample acquired every $0.005 \lambda$. This experiment was then repeated for motion at $30 \mathrm{~cm} / \mathrm{s}$ over a distance of $9 \mathrm{~m}$, with a sample acquired every $0.06 \lambda$. Comparison of these results revealed that the channel variation could be captured with insignificant error using the higher receiver speed.

The array orientations, receiver motion path (relative to Fig. 5), and metrics resulting from the acquired data are listed in Table II. The metrics confirm that this environment has relatively slow temporal variation. The eigenvalue variation indicates that the MAC should optimally adapt at a rate less than once per wavelength. However, since the RCD capacity degradation is significant for distances on the order of $\lambda / 4$, the receive PHY must adapt at a higher rate.

The results also show that the monopole antennas exhibit higher channel variation than the patch antennas. This concept is reinforced by Fig. 6, which plots a sample time evolution of the first four eigenvalues for Set 1 (patches) and Set 4 (monopoles). The rapid variation observed for the monopole array is likely due to the wide angular spread of arrivals collected by omnidirectional elements. In contrast, the dual-
TABLE II

ArRay Configurations AND MEtrics For DT FiEld (ENVIRONMENT 1)

\begin{tabular}{|l|rrr|rr|}
\hline Set & 1 & 2 & 3 & 4 & 5 \\
\hline Arrays & $8 \mathrm{P}$ & $8 \mathrm{P}$ & $8 \mathrm{P}$ & $7 \mathrm{M}$ & $7 \mathrm{M}$ \\
$\mathrm{Tx}$ & $\leftarrow$ & $\leftarrow$ & $\uparrow$ & $\rightarrow$ & $\uparrow$ \\
$\mathrm{Rx}$ & $\leftarrow$ & $\downarrow$ & $\leftarrow$ & $\leftarrow$ & $\downarrow$ \\
Path & $\downarrow$ & $\longrightarrow$ & $\downarrow$ & $\leftarrow$ & $\downarrow$ \\
\hline ELCR $_{1}$ & 0.10 & 0.37 & 0.10 & 0.25 & 0.45 \\
ELCR $_{2}$ & 0.08 & 0.38 & 0.27 & 0.55 & 0.52 \\
$\mathrm{ELCR}_{3}$ & 0.42 & 0.47 & 0.33 & 0.73 & 0.38 \\
\hline $\mathrm{ES}$ & 8.7 & 9.3 & 7.4 & 10.9 & 9.1 \\
\hline$C_{\mathrm{WF}}$ & 17.7 & 17.2 & 19.8 & 16.8 & 16.9 \\
$C_{\mathrm{UT}}$ & 15.0 & 14.7 & 17.6 & 14.3 & 14.9 \\
\hline$d_{T} / \lambda$ & $>10$ & $>10$ & 1.80 & $>10$ & $>10$ \\
$d_{R} / \lambda$ & 0.35 & 0.15 & 0.25 & 0.15 & 0.20 \\
\hline
\end{tabular}

(a)

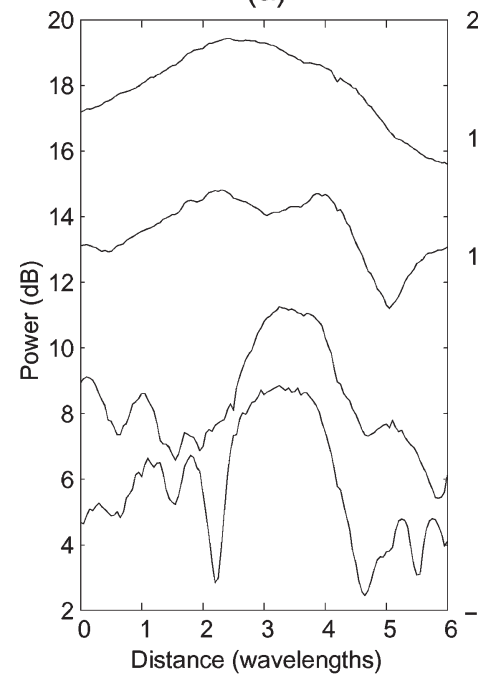

(b)

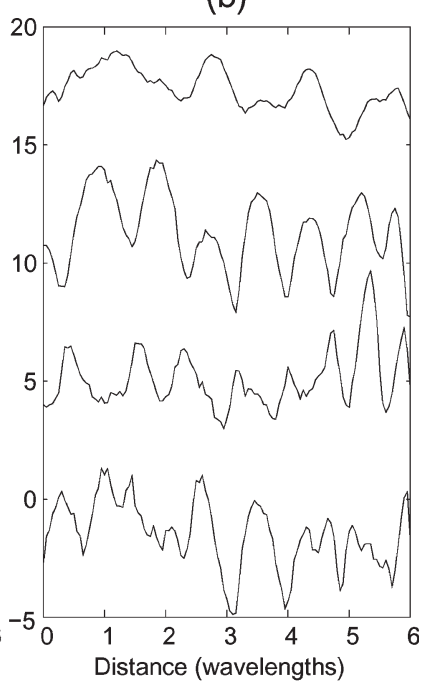

Fig. 6. Sample temporal evolution of eigenvalues from Environment 1 for (a) Set 1 with patches and (b) Set 6 with monopoles.

polarized patches have more directive patterns, resulting in reduced sensitivity to position.

Fig. 7 plots the eigenvalue probability density functions (pdfs) for channels obtained with four vertical patch elements and four monopoles (from Sets 1 and 4). These results show that the eigenvalues (and therefore capacities) are nearly identical for the two antennas. This observation suggests that antennas with more spatial selectivity may be advantageous for MIMO systems in environments with high mobility since they offer high capacity while exhibiting lower temporal variability.

Fig. 8 plots spatial spectra for Sets 1 and 2. The spectra for Set 1 are narrow due to a dominant reflection from the building (Conference Center) to the west, corresponding to fairly slow variation as quantified by the metrics. In contrast, for Set 2 , the receiver points south toward more distant buildings, and since no single-bounce propagation mechanism is present, the spectra are much wider, resulting in faster channel variation.

\section{B. Clyde Building (CB) Trees}

In the second measurement campaign, the system nodes were placed in the midst of sparse trees near the $\mathrm{CB}$, as shown in Fig. 9. The environment was influenced by occasional passing pedestrians. The transmitter was placed in front of the building 


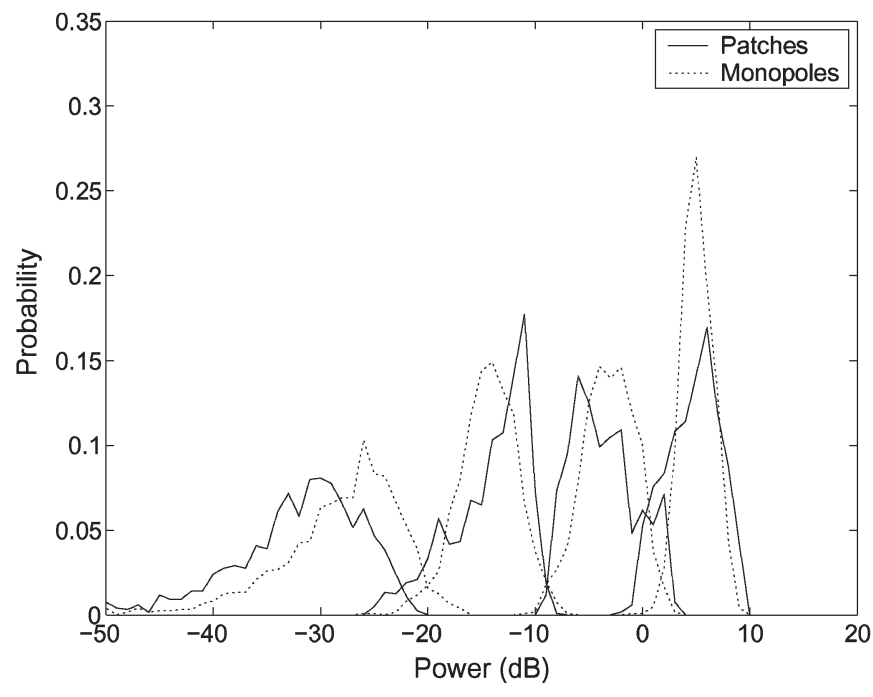

Fig. 7. Eigenvalue pdfs of four patches (Set 1) compared with four monopole elements (Set 6).

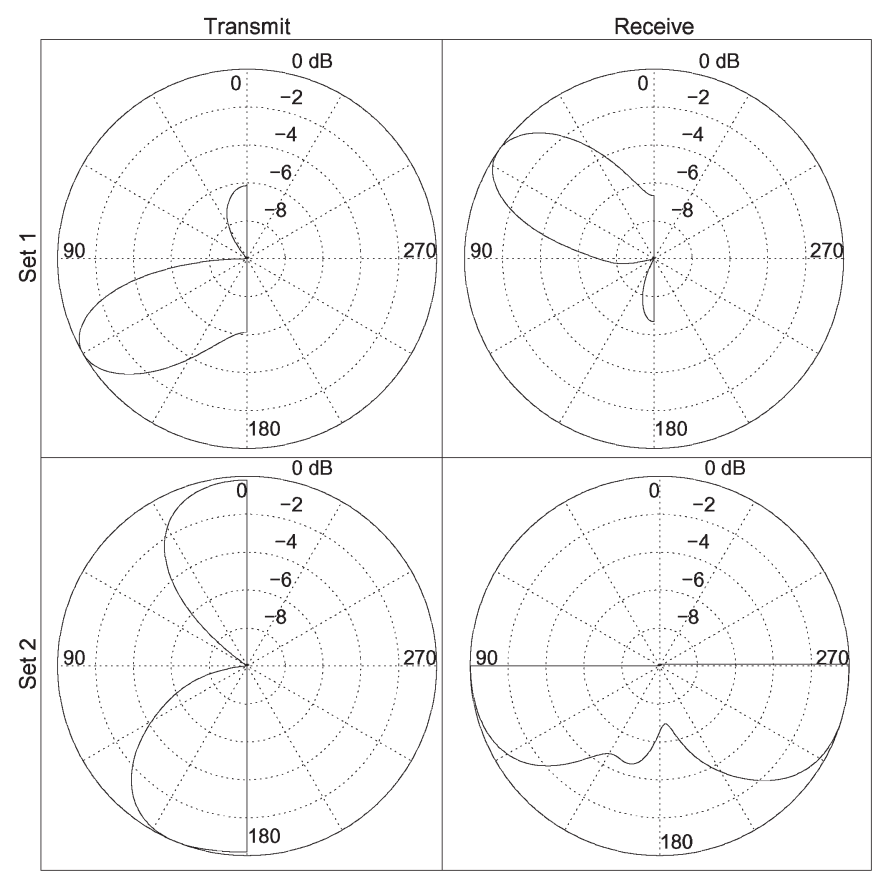

Fig. 8. Transmit and receive spectra for data sets 1 and 2, indicating much higher angular spread for set 2 .

behind two trees, whereas the receiver assumed a number of possible positions. For each measurement, the receiver was either stationary (to observe the effect of pedestrians) or moved $9 \mathrm{~m}$ along a straight path. The crossing rates for the stationary measurements were all zero except for the third eigenvalue on a single data set. Therefore, we will assume the effect of pedestrians to be negligible and focus on the moving cases.

Table III summarizes the array configurations and metrics in this environment. These data exhibit even less variation than the DT field measurements, particularly for patch arrays. As with DT field, the location with the highest variation (Set 3) has very wide angular spread of multipath at transmit and receive. For sets with very low variation (Sets 4 and 8), the spatial spectra are much narrower, suggesting a strong nonfading path through

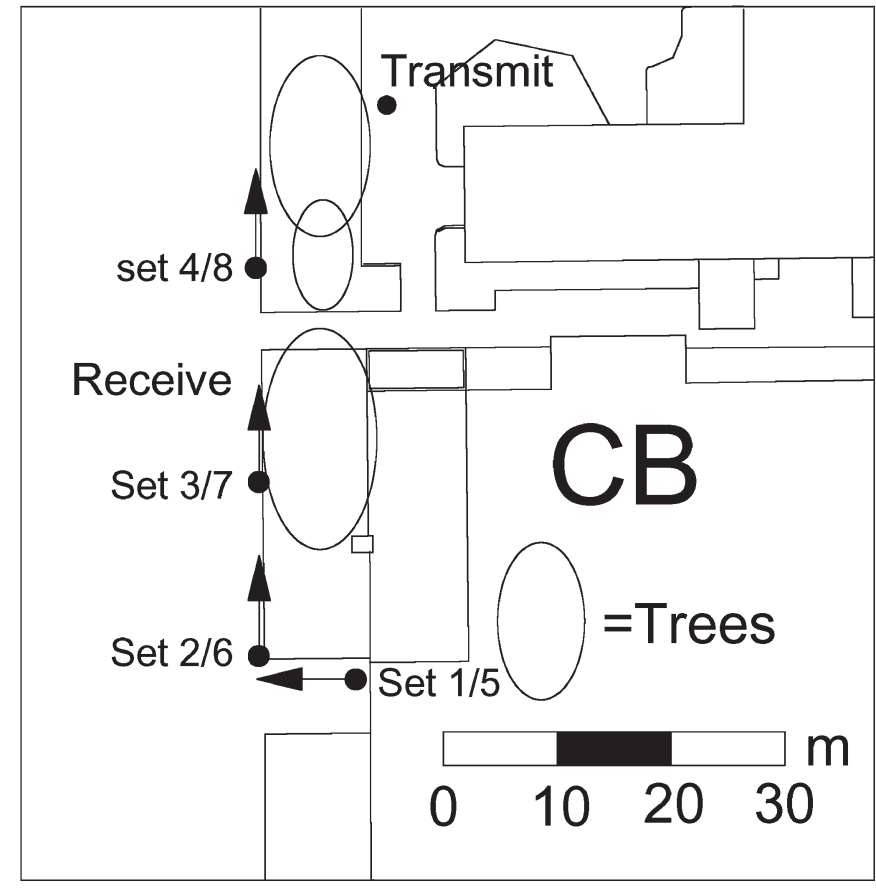

Fig. 9. Map of Environment 2 (CB Trees). Transmitter was placed behind sparse trees, and the receiver assumed three different positions on the opposite side of the trees. Distances are in meters.

TABLE III

Array Configurations and Metrics For CB Trees (ENVIRONMENT 2)

\begin{tabular}{|l|rrrr|rrrr|}
\hline Set & 1 & 2 & 3 & 4 & 5 & 6 & 7 & 8 \\
\hline Arrays & $8 \mathrm{M}$ & $8 \mathrm{M}$ & $8 \mathrm{M}$ & $8 \mathrm{M}$ & $8 \mathrm{P}$ & $8 \mathrm{P}$ & $8 \mathrm{P}$ & $8 \mathrm{P}$ \\
$\mathrm{Tx}$ & $\swarrow$ & $\swarrow$ & $\swarrow$ & $\swarrow$ & $\swarrow$ & $\swarrow$ & $\swarrow$ & $\swarrow$ \\
$\mathrm{Rx}$ & $\nearrow$ & $\nearrow$ & $\nearrow$ & $\nearrow$ & $\uparrow$ & $\uparrow$ & $\uparrow$ & $\uparrow$ \\
\hline $\mathrm{ELCR}_{1}$ & 0.27 & 0.17 & 0.97 & 0.17 & 0.02 & 0.10 & 0.06 & 0.03 \\
$\mathrm{ELCR}_{2}$ & 0.65 & 0.08 & 0.37 & 0.16 & 0.05 & 0.05 & 0.07 & 0.14 \\
$\mathrm{ELCR}_{3}$ & 0.54 & 0.25 & 0.38 & 0.28 & 0.31 & 0.20 & 0.43 & 0.23 \\
\hline $\mathrm{ES}$ & 11.4 & 6.0 & 5.6 & 6.9 & 10.2 & 5.4 & 8.5 & 10.9 \\
\hline$C_{\mathrm{WF}}$ & 16.2 & 20.1 & 19.6 & 20.3 & 16.4 & 21.0 & 17.6 & 17.7 \\
$C_{\mathrm{UT}}$ & 13.9 & 18.0 & 17.6 & 18.4 & 14.1 & 19.4 & 15.6 & 15.5 \\
\hline$d_{T} / \lambda$ & 3.25 & $>10$ & 4.00 & 4.45 & $>10$ & 3.60 & $>10$ & 5.55 \\
$d_{R} / \lambda$ & 0.20 & 0.25 & 0.15 & 0.30 & 0.35 & 0.70 & 1.45 & 1.45 \\
\hline
\end{tabular}

the trees. Given the variation rates, it appears reasonable that the MAC adapt at a rate less than once per wavelength. The receive PHY, however, may still need to update reception weights on the $\lambda / 4$ scale.

\section{Coal Yard}

The third environment consisted of a parking lot with parked cars surrounded by many metal buildings, as depicted in Fig. 10. The transmitter assumed one of three possible lettered positions in the diagram, and the receiver assumed one of two possible numbered positions. The receiver was either stationary or moved along a straight $9-\mathrm{m}$ path at $30 \mathrm{~cm} / \mathrm{s}$. For the stationary measurements, the crossing rates were again almost always zero, showing that any cars moving in the channel had a nearly negligible effect.

Table IV summarizes the measurement parameters and metrics for the moving cases. The metrics reveal that the variation is only slightly higher (on average) than the variation seen at the DT field. However, certain positions (Set 1, for example) 


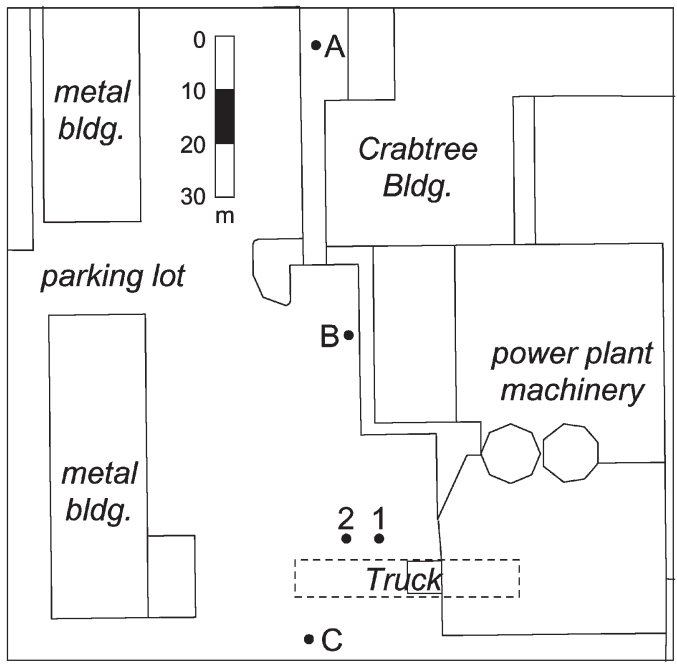

Fig. 10. Map of Environment 3 (Coal Yard). Transmitter was at one of the lettered positions, whereas the receiver was placed at one of the numbered positions. For Sets 7 and 8, a coal truck was parked in the coal yard as indicated. Distances are in meters.

TABLE IV

ARRAY CONFIGURATIONS AND METRICS FOR COAL YARD (ENVIRONMENT 3)

\begin{tabular}{|l|rr|rrrrrr|}
\hline Set & 1 & 2 & 3 & 4 & 5 & 6 & 7 & 8 \\
\hline Arrays & $8 \mathrm{M}$ & $8 \mathrm{M}$ & $8 \mathrm{P}$ & $8 \mathrm{P}$ & $8 \mathrm{P}$ & $8 \mathrm{P}$ & $8 \mathrm{P}$ & $8 \mathrm{P}$ \\
Pos & $\mathrm{A} 1$ & $\mathrm{~A} 1$ & $\mathrm{~A} 1$ & $\mathrm{~A} 1$ & $\mathrm{~B} 1$ & $\mathrm{~B} 1$ & $\mathrm{C} 2$ & $\mathrm{C} 1$ \\
Tx & $\rightarrow$ & $\rightarrow$ & $\leftarrow$ & $\leftarrow$ & $\leftarrow$ & $\leftarrow$ & $\swarrow$ & $\swarrow$ \\
$\mathrm{Rx}$ & $\swarrow$ & $\nwarrow$ & $\downarrow$ & $\leftarrow$ & $\downarrow$ & $\leftarrow$ & $\rightarrow$ & $\leftarrow$ \\
Path & $\downarrow$ & $\leftarrow$ & $\downarrow$ & $\leftarrow$ & $\downarrow$ & $\leftarrow$ & $\rightarrow$ & $\leftarrow$ \\
\hline ELCR $_{1}$ & 0.63 & 0.34 & 0.17 & 0.25 & 0.36 & 0.03 & 0.30 & 0.08 \\
ELCR $_{2}$ & 0.41 & 0.34 & 0.42 & 0.49 & 0.36 & 0.23 & 0.40 & 0.12 \\
ELCR $_{3}$ & 0.96 & 0.36 & 0.28 & 0.55 & 0.30 & 0.19 & 0.40 & 0.20 \\
\hline ES & 9.2 & 7.5 & 7.2 & 10.1 & 6.3 & 9.4 & 6.7 & 6.1 \\
\hline$C_{\mathrm{WF}}$ & 18.8 & 18.4 & 19.5 & 17.8 & 20.4 & 18.2 & 20.7 & 19.3 \\
$C_{\mathrm{UT}}$ & 16.7 & 16.1 & 17.6 & 15.4 & 18.5 & 15.5 & 19.0 & 17.1 \\
\hline$d_{T} / \lambda$ & 8.25 & $>10$ & $>10$ & 4.30 & 2.25 & $>10$ & 1.15 & $>10$ \\
$d_{R} / \lambda$ & 0.10 & 0.20 & 0.20 & 0.30 & 0.10 & 0.45 & 0.10 & 0.55 \\
\hline
\end{tabular}

exhibit much more variation, revealing that the same physical scenario can produce channels with both high and low variations. Additional analysis not included here again reveals that Sets 3,5, and 7, which represent the channels with high time variation, are characterized by larger angular spread as compared to Sets 4,6 , and 8 .

\section{D. $C B$ Corridor}

Fig. 11 shows a map of measurements taken when the nodes sat in corridors between buildings. For all sets, the transmitter assumed a fixed position and the receiver was either moved along a $27-\mathrm{m}$ path or rotated two times.

Large-scale movement and rotation led to much more pronounced changes in the channel than had been seen previously. However, large transitions led mainly to changes in the path loss as opposed to changes in the amount of multipath present. Fig. 12 shows the spatial spectra for Set 1 as the receiver is moved along a corridor between the buildings. Since the path is quite long, the spatial spectra have been computed separately at the beginning (B), middle (M), and end (E) of the path. Interestingly, when the receiver is the most obscured (B), the

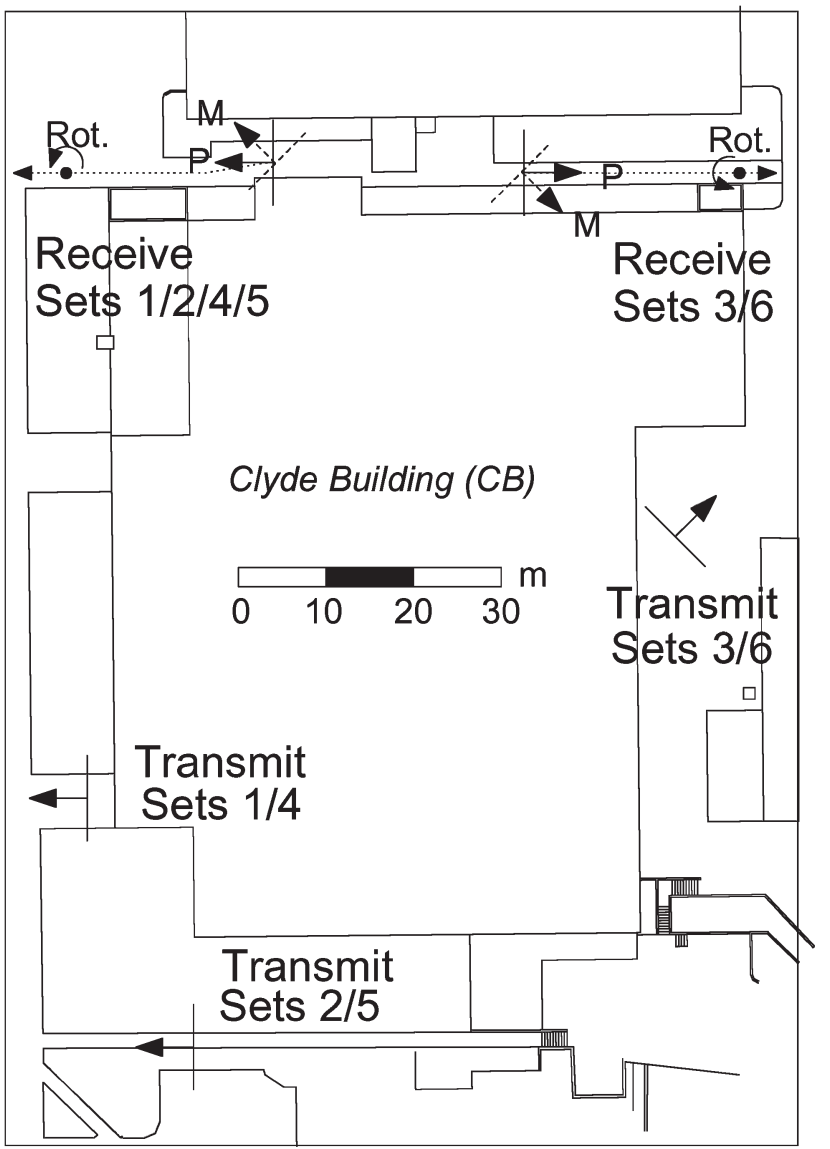

Fig. 11. Map of Environment 4 (CB Corridor) showing positions and orientations of arrays. The transmitter was at three different locations, and the receiver was moved along a $27-\mathrm{m}$ path (dotted line) in a corridor between two buildings or rotated (Rot.). Patches were used for Sets 1-3 and monopoles for Sets 4-6. The orientation for the receiver monopole array (M) was shifted by $45^{\circ}$ relative to the patches $(\mathrm{P})$.

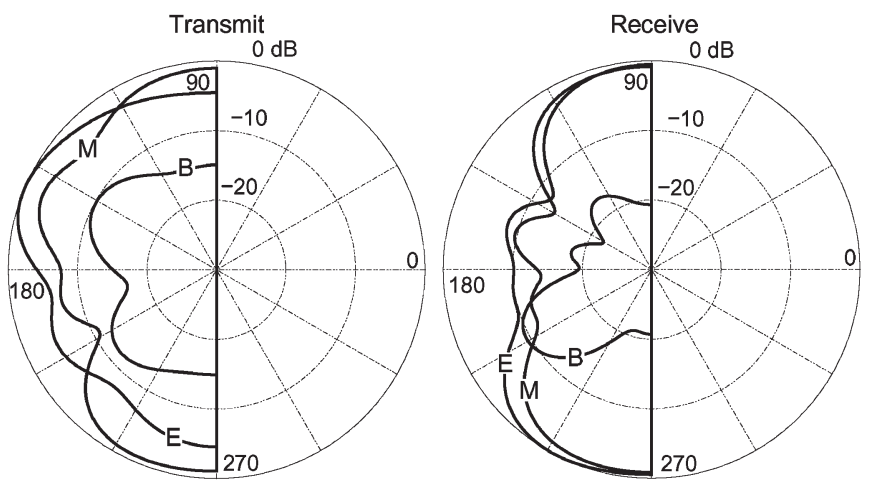

Fig. 12. Transmit/receive spectra for Environment 4, Set 1 for the beginning (B), middle (M), and end (E) of the path.

spectra look the most directional, and when the receiver moves out into the open, the spectra become wider. These results can possibly be explained by a waveguiding phenomenon, since a waveguide would exhibit only a few strong propagation directions.

The effect of the large-scale movement and rotation on the eigenvalues is depicted in Fig. 13 for Set 1. These results show a change in the overall eigenvalue levels (due to path loss) rather than a change in the ES. Table $\mathrm{V}(\mathrm{a})$ and (b) summarizes the 
(a)

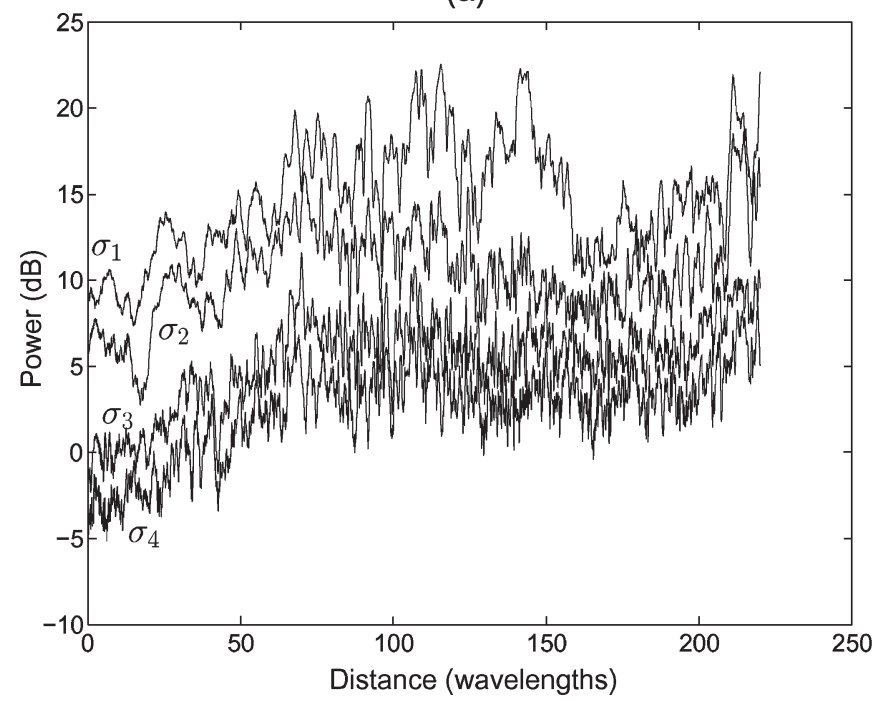

(b)

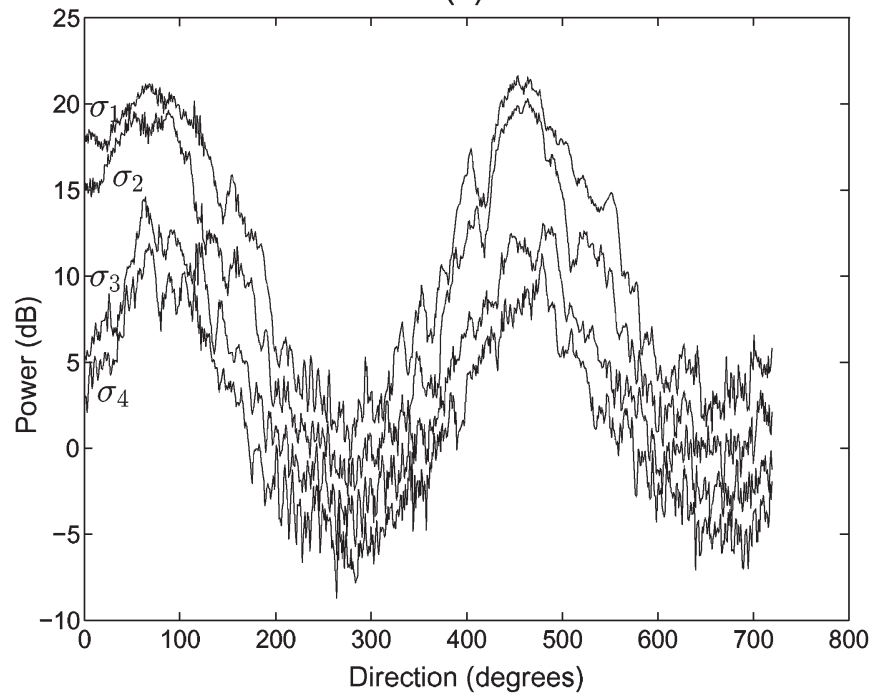

Fig. 13. Variation of the first four eigenvalues for Environment 4, Set 1 for (a) receiver movement and (b) receiver rotation, indicating mainly changes in the path loss.

metrics for this location for moving and rotating measurements. For rotating measurements, ELCR is measured in number of eigenvalue crossings per $10^{\circ}$ of rotation, and $d_{R}$ is measured in degrees. Metrics for the moving case show that the rate of variation here was lower than that for DT field. Although surprising, perhaps the waveguiding effect of the buildings partially accounts for this effect. Variation of the channels for patch antennas and monopoles is quite similar, in contrast to other measurements where the monopoles exhibit higher variation. This can be explained by the fact that the directional arrays were usually pointed where the maximum power transfer would occur (i.e., down the corridor), and therefore, it is unlikely that the monopoles would collect significantly more multipath than the patches.

\section{E. Discussion}

Table VI summarizes average values of the metrics for monopole and patch arrays across all of the environments in
TABLE V

METRICS FOR CB CORRIDOR (ENVIRONMENT 4) (a) LARGE-SCALE MOVEMENT (b) ROTATION (ELCR IN CROSSING 110)

(a)

\begin{tabular}{|l|rrr|rrr|}
\hline Set & 1 & 2 & 3 & 4 & 5 & 6 \\
\hline Arrays & $8 \mathrm{P}$ & $8 \mathrm{P}$ & $8 \mathrm{P}$ & $8 \mathrm{M}$ & $8 \mathrm{M}$ & $8 \mathrm{M}$ \\
\hline ELCR $_{1}$ & 0.10 & 0.17 & 0.01 & 0.04 & 0.23 & 0.03 \\
ELCR $_{2}$ & 0.23 & 0.39 & 0.03 & 0.03 & 0.20 & 0.22 \\
ELCR $_{3}$ & 0.23 & 0.83 & 0.02 & 0.06 & 0.36 & 0.20 \\
\hline $\mathrm{ES}$ & 9.8 & 15.0 & 7.6 & 5.9 & 7.4 & 6.2 \\
\hline$C_{\mathrm{WF}}$ & 17.4 & 13.4 & 13.7 & 14.5 & 17.0 & 17.4 \\
$C_{\mathrm{UT}}$ & 15.4 & 10.3 & 11.7 & 12.1 & 14.7 & 15.5 \\
\hline$d_{T} / \lambda$ & 2.45 & $>10$ & $>10$ & $>10$ & $>10$ & 4.15 \\
$d_{R} / \lambda$ & 0.25 & 0.30 & 0.45 & 0.40 & 0.15 & 0.25 \\
\hline
\end{tabular}

(b)

\begin{tabular}{|l|rrr|rrr|}
\hline Set & 1 & 2 & 3 & 4 & 5 & 6 \\
\hline Arrays & $8 \mathrm{P}$ & $8 \mathrm{P}$ & $8 \mathrm{P}$ & $8 \mathrm{M}$ & $8 \mathrm{M}$ & $8 \mathrm{M}$ \\
\hline ELCR $_{1}$ & 0.22 & 0.16 & 0.12 & 0.75 & 0.50 & 0.56 \\
ELCR $_{2}$ & 0.72 & 0.09 & 0.12 & 1.06 & 0.97 & 1.28 \\
ELCR $_{3}$ & 0.41 & 0.16 & 0.44 & 1.62 & 1.84 & 2.22 \\
\hline ES & 8.1 & 7.4 & 12.1 & 6.6 & 11.2 & 8.7 \\
\hline$C_{\mathrm{WF}}$ & 15.3 & 13.5 & 12.7 & 20.0 & 17.7 & 19.0 \\
$C_{\mathrm{UT}}$ & 13.3 & 11.1 & 10.4 & 17.9 & 15.2 & 17.0 \\
\hline$d_{R}$ & $3^{\circ}$ & $6^{\circ}$ & $6^{\circ}$ & $3^{\circ}$ & $3^{\circ}$ & $3^{\circ}$ \\
\hline
\end{tabular}

TABLE VI

Average Metrics Versus Environment

\begin{tabular}{|l|rrrr|rrrr|}
\hline & \multicolumn{4}{|c|}{ Monopoles } & \multicolumn{4}{|c|}{ Patches } \\
Env. & 1 & 2 & 3 & 4 & 1 & 2 & 3 & 4 \\
\hline ELCR $_{1}$ & 0.35 & 0.39 & 0.49 & 0.10 & 0.19 & 0.05 & 0.20 & 0.09 \\
ELCR $_{2}$ & 0.53 & 0.31 & 0.38 & 0.15 & 0.24 & 0.08 & 0.33 & 0.21 \\
ELCR $_{3}$ & 0.56 & 0.36 & 0.66 & 0.21 & 0.41 & 0.29 & 0.32 & 0.36 \\
\hline ES & 10.0 & 7.5 & 8.4 & 6.5 & 8.4 & 8.7 & 7.6 & 10.8 \\
\hline$C_{\mathrm{WF}}$ & 16.9 & 19.0 & 18.6 & 16.3 & 18.2 & 18.2 & 19.3 & 14.8 \\
$C_{\mathrm{UT}}$ & 14.6 & 17.0 & 16.4 & 14.1 & 15.8 & 16.2 & 17.2 & 12.5 \\
\hline$d_{T} / \lambda$ & $>10$ & 5.42 & 9.12 & 8.05 & 7.27 & 7.29 & 6.28 & 7.48 \\
$d_{R} / \lambda$ & 0.18 & 0.23 & 0.15 & 0.27 & 0.25 & 0.99 & 0.28 & 0.33 \\
\hline
\end{tabular}

this paper. This paper indicates that the physical scattering environment has much less of an effect on the time variability of channels than the array configurations and orientations of the MIMO system. Dual-polarized directional patch antennas produced channels with considerably lower temporal variation than omnidirectional monopoles, indicating that spatially selective elements may be advantageous for highly mobile systems. This idea is also supported by the fact that throughout the measurements, a very strong correlation between the angular spread of spatial spectra and the temporal variation was evident.

The measurements also indicate the level of adaptation required of advanced mobile MIMO architectures. Table VI gives average eigenvalue crossing rates on the order of $0.4 / \lambda$ and $0.2 / \lambda$ for monopoles and patches, respectively, indicating that an advanced adaptive MIMO MAC/PHY would need to update its modulation and rate a few times per wavelength. On the other hand, values for $d_{R}$ are about $0.2 \lambda$ and $0.5 \lambda$ for monopoles and patches, respectively, suggesting that training must be performed rapidly at the receive PHY to achieve high capacity. Although $d_{T}$ can be quite large, indicating that transmit CSI is useful for long distances, the increase in capacity when the transmitter knows the channel was fairly modest for our measured channels. 


\section{CONCLUSION}

This paper presents MIMO channel measurements conducted in an outdoor campus environment at $2.45 \mathrm{GHz}$ and analyzes the data behavior in terms of channel temporal variation. A number of useful metrics are developed to quantify MIMO time variation and its effect on system performance. The results indicate that rates of system adaptation are on the order of $\lambda / 4$ for the PHY and $1 \lambda$ for higher level adaptation of the transmission rate and modulation. The analysis should be useful for the design of MIMO systems for mobile environments.

\section{REFERENCES}

[1] M. A. Jensen and J. W. Wallace, "A review of antennas and propagation for MIMO wireless communications," IEEE Trans. Antennas Propag., vol. 52, no. 11, pp. 2810-2824, Nov. 2004.

[2] L. Zheng and D. N. C. Tse, "Diversity and multiplexing: A fundamental tradeoff in multiple antenna channels," IEEE Trans. Inf. Theory, vol. 49, no. 5, pp. 1073-1096, May 2003.

[3] A. J. Goldsmith, S. A. Jafar, N. Jindal, and S. Vishwanath, "Capacity limits of MIMO channels," IEEE J. Sel. Areas Commun., vol. 21, no. 5, pp. 684-702, Jun. 2003.

[4] B. M. Hochwald and W. Sweldens, "Differential unitary space-time modulation," IEEE Trans. Commun., vol. 48, no. 12, pp. 2041-2052, Dec. 2000.

[5] B. Hassibi and B. M. Hochwald, "How much training is needed in multiple-antenna wireless links?" IEEE Trans. Inf. Theory, vol. 49, no. 4, pp. 951-963, Apr. 2003.

[6] J. Baltersee, G. Fock, and H. Meyr, "Achievable rate of MIMO channels with data-aided channel estimation and perfect interleaving," IEEE J. Sel. Areas Commun., vol. 19, no. 12, pp. 2358-2368, Dec. 2001.

[7] D. P. McNamara, M. A. Beach, and P. N. Fletcher, "Experimental investigation of the temporal variation of MIMO channels," in Proc. IEEE 54th Veh. Technol. Conf., Atlantic City, NJ, Oct. 7-11, 2001, pp. 1063-1067.

[8] H. Xu, M. Gans, D. Chizhik, J. Ling, P. Wolniansky, and R. Valenzuela, "Spatial and temporal variations of MIMO channels and impacts on capacity," in Proc. IEEE Int. Conf. Commun., New York, Apr. 28-May 2, 2002, vol. 1, pp. 262-266.

[9] V. Pohl, P. H. Nguyen, V. Jungnickel, and C. von Helmolt, "How often channel estimation is needed in MIMO systems," in Proc. IEEE Global Telecommun. Conf., San Francisco, CA, Dec. 1-5, 2003, vol. 2, pp. 814-818.

[10] J. W. Wallace, M. A. Jensen, A. L. Swindlehurst, and B. D. Jeffs, "Experimental characterization of the MIMO wireless channel: Data acquisition and analysis," IEEE Trans. Wireless Commun., vol. 2, no. 2, pp. 335-343, Mar. 2003.

[11] J. Maurer, C. Waldschmidt, T. Kayser, and W. Wiesbeck, "Characterisation of the time-dependent urban MIMO channel in FDD communication systems," in Proc. IEEE 57th Veh. Technol. Conf., Jeju, Korea, Apr. 22-25, 2003 , vol. 4 , pp. $544-548$.

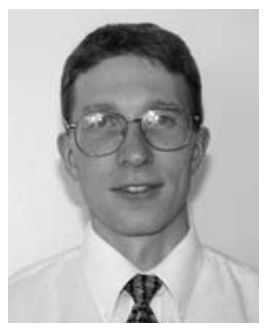

Jon W. Wallace (S'99-M'03) received the B.S. (summa cum laude) and $\mathrm{Ph} . \mathrm{D}$. degrees in electrical engineering from Brigham Young University (BYU), Provo, UT, in 1997 and 2002, respectively.

From 1995 to 1997, he was an Associate at Novell, Inc., Provo. During 1997, he was a member of Technical Staff at Lucent Technologies, Denver, CO. He received the National Science Foundation Graduate Fellowship in 1998 and was a Graduate Research Assistant at BYU until 2002. From 2002 to 2003, he was with the Mobile Communications Group, Vienna University of Technology, Vienna, Austria, where he pursued a collaborative research in the area of wireless channel measurement and modeling. From 2003 to 2006, he was a Research Associate at the Wireless Communications Laboratory, BYU, where he developed platforms for wideband MIMO channel sounding and real-time space-time coding. He is currently an Assistant Professor in the School of Engineering and Science, Jacobs University Bremen (formerly International University of Bremen), Bremen, Germany. His research interests include wireless channel sounding and modeling, real-time MIMO implementation, optical device modeling, and remote sensing.

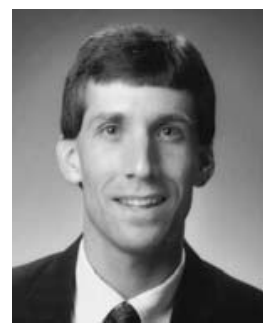

Michael A. Jensen (S'93-M'95-SM'01) received the B.S. (summa cum laude) and M.S. degrees in electrical engineering from Brigham Young University (BYU), Provo, UT, in 1990 and 1991, respectively, and the Ph.D. degree in electrical engineering from the University of California, Los Angeles (UCLA), in 1994.

From 1989 to 1991, he was a Graduate Research Assistant in the Lasers and Optics Laboratory, BYU. From 1991 to 1994, he was a Graduate Student Researcher in the Antenna Laboratory, UCLA. Since 1994, he has been with the Electrical and Computer Engineering Department, BYU, where he is currently a Professor and Department Chair. His main research interests include antennas and propagation for personal communications, microwave circuit design, radar remote sensing, numerical electromagnetics, and optical fiber communications.

Dr. Jensen is a member of Eta Kappa Nu and Tau Beta Pi. He is currently a member of the Administrative Committee and the Joint Meetings Committee for the IEEE Antennas and Propagation Society, is an Associate Editor for the IEEE TRANSACTIONS ON ANTENNAS AND PROPAGATION, and has served the society as Vice Chair and Technical Program Chair for several symposia. He is the recipient of the H. A. Wheeler Paper Award in the IEEE TRANSACTIONS ON ANTENNAS AND PRopagation in 2002 and the Best Student Paper Award at the 1994 IEEE International Symposium on Antennas and Propagation.

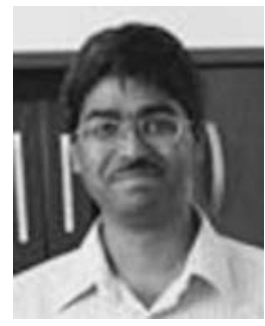

Ajay Gummalla (S'96-M'00) received the B.Tech. degree from Indian Institute of Technology, Madras, India, in 1996 and the M.S. and Ph.D. degrees in electrical engineering from Georgia Institute of Technology, Atlanta, in 2000.

He has worked extensively in broadband wireless and wired communication systems. He recently joined Rayspan Corporation, San Diego, CA, as the Director of Communication Systems. Prior to this, he was a Principle Member of Technical Staff at San Diego Research Center, where he led the research, design, development, and successful demonstration of Mobile MIMO technology. Previously, he made significant contributions to the IEEE 802.3ah Ethernet in the First Mile study group and was a key contributor in the development of "Propane" technology at Broadcom (Digital Furnace). He has authored numerous journal and conference papers. He is the holder of eight patents and is a Coinventor on 12 patent applications. His current research interests include MIMO wireless communications, wireless mesh/ad hoc networks, and broadband access technologies.

Harry B. Lee (S'61-M'63) was born in Chicago, IL, on July 27, 1935. He received the $\mathrm{Ph} . \mathrm{D}$. degree in electrical engineering from Massachusetts Institute of Technology (MIT), Cambridge, in 1962.

From 1963 to 1967, he was an Assistant Professor of Electrical Engineering at MIT. He is currently with San Diego Research Center, Inc., San Diego, CA. He has cofounded three successful high-tech companies, namely: 1) Applicon (1969), 2) Atlantic Aerospace Electronics Corporation (1985), and 3) San Diego Research Center, Inc. (2001). His professional interests include geolocation, direction finding, MIMO, LPD/AJ, and adaptive techniques.

Dr. Lee is the recipient of the IEEE Browder J. Thompson Award in 1964 for the best paper by an author under age 30 and the IEEE Russel Baker Award in 1968 for the best paper by an author of any age. 